\title{
NOTA PRELIMINAR SOBRE A COLEÇÃO MALACOLÓGICA "ELISEO DUARTE IV"'
}

\author{
Paulo Tadeu Campos Lopes ${ }^{2}$ \\ Marcia Moccellin Raymundo ${ }^{2}$ \\ Álvaro Luís Müller da Fonseca ${ }^{2}$ \\ Giovana Bervian ${ }^{3}$ \\ José Willibaldo Thomé ${ }^{4}$
}

Esta é a quarta comunicação (MENEZES \& THOMÉ, 1985; PICORAL \& THOMÉ, 1987; PICORAL et al., 1989) sobre a coleção malacológica "Eliseo Duarte" e apresenta uma revisão dos lotes catalogados até o presente. A coleção foi adquirida pelo CNPq e encontra-se em custódia da Fundação Zoobotânica do Rio Grande do Sul.

A classificação utilizada é a de TAYLOR \& SOHL (1962) para a classe Gastropoda, MOORE (1969) para a classe Bivalvia e RIOS (1985) para as Classes Polyplacophora, Scaphopoda e Cephalopoda.

O ritmo de catalogação foi praticamente triplicado, devido ao alto grau de especialização atingido pelos bolsistas.

Os lotes catalogados até agora são em número de 8.275 (um acréscimo de 4.561 lotes desde a última nota), com 47.239 exemplares. Isto corresponde a cerca de metade da coleção. 0 material provém de todo o mundo, achando-se distribuído pelas seguintes regiões zoogeográficas: Holártica $=3.277$ lotes $(39,6 \%)$; Neotropical $=2.598$ lotes $(31,4 \%)$; Australiana $=902$ lotes $(10,9 \%)$; Oriental $=662$ lotes $(8,0 \%)$; Etiópica $=447$ lotes $(5,4 \%)$; Antártica $=223$ lotes $(2,7 \%)$; Oceânica $=83$ lotes $(1,0 \%)$; sem procedência $=83$ lotes $(1,0 \%)$. A presente listagem compreende 185 famílias, das quais 2 são poliplacóforos; 150 são gastrópodes; 30 são bivalves; 1 é escafópode e 2 são cefalópodes.

A divulgação da listagem destina-se ao conhecimento da disponibilidade de material na coleção, para uso de cientistas e pesquisadores credenciados.

Listagem:

Filo MOLLUSCA

Classe POLYPLACOPHORA

Ordem NEOLORICATA

Subordem ISCHNOCHITONINA

Lotes Espécimes

Ischnochitonidae

Mopaliidae

1. Apresentado no XVHI Congresso Brasileiro de Zoologia, Salvador, BA.

2. Bolsistas de Inic iação Científica do CNPq.

3. Bolsista de Iniciação Científica da FAPERGS.

4. Professor da PUCRS/Presidente da FZB-RS - Av. Dr. Salvador França, no 1.427 (Jardim Botânico) -90.610 , Porto Alegre, RS 
Subclasse STREPTONEURA

Ordem ARCHAEOGASTROPODA

Pleurotomariidae

Haliotidae

Fissurellidae

Acmaeidae

Patellidae

Lepetidae

Trochidae

Turbinidae

Skeneidae

Phasianellidae

Neritidae

Helicinidae

Ordem MESOGASTROPODA

Cyclophoridae

Poteriidae

Pupinidae

Cochlostomatidae

Viviparidae

Ampullariidae

Lavigeriidae

Valvatidae

Lacunidae

Littorinidae

Pomatiasidae

Chondropomidae

Hydrobiidae

Truncatellidae

Stenothyridae

Bithyniidae

Rissoidae

Assimineidae

Amastridae

Cionellidae

Pyramidulidae

Vertiginidae

Orculidae

Chondrinidae

Pupillidae

Valloniidae

Pleurodiscidae

Enidae

Subordem MESURETHRA

Clausiliidae 
Vol. 7(1-2), 1990

Strophoceilidae

Megalobulimidae

Subordem HETERURETHRA

Succineidae

Subordem SIGMURETHRA

Ferrussaciidae

Subulinidae

Achatinidae

Streptaxidae 35

Acavidae

Haplotrematidae

Rhytididae

Bulimulidae

673

Odontostomidae

145

Orthalicidae

61

115

Urocoptidae

Cerionidae

Endodontidae

132

Vitrinidae

Zonitidae

Parmacellidae

Limacidae

Trigonochlamydidae

188

Systrophiidae

Euconulidae

Heliocarionidae

Ariophantidae

Urocyclidae

Testacellidae

Vitrinellidae

Architectonicidae

Turritellidae

Mathildidae

Vermetidae

Caecidae

19

10

42

Thiaridae

Melanopsidae

Pleuroceridae

Planaxidae

Modulidae

Potamididae

Cerithiidae

Epitoniidae

Janthinidae

Eulimidae

Struthiolariidae 
Hipponicidae

10

Trichotropidae

Capulidae

Calyptraeidae

Xenophoridae

Lamellariidae

Eratoidae

Cypraeidae

Ovulidae

Naticidae

Cassididae

Cymatiidae

Bursidae

Tonnidae

Ficidae

Ordem NEOGASTROPODA

Subordem STENOGLOSSA

Muricidae

Magilidae

Pyrenidae

Buccinidae

Melongenidae

Nassariidae

Fasciolariidae

Olividae

Vasidae

Harpidae

Volutidae

Cancellaridae

Marginellidae

Costellariidae

Subordem TOXOGLOSSA

Mitridae

Turridae

Conidae

Terebridae

Subclasse EUTHYNEURA

Ordem ENTOMOTAENIATA

Pyramidellidae

Ordem CEPHALASPIDEA

Acteonidae

Ringiculidae

Hydatinidae

Scaphandridae

Philinidae

Bullidae

Atyidae

Retusidae

53

8


Vol. 7(1-2), 1990

Ordem BASOMMATOPHORA

Melampidae

Trimusculidae

Siphonariidae

Amphibolidae

Ellobiidae

Chilinidae

272

Acroloxidae

Lymnaeidae

Ancylidae

Planorbidae

Physidae

Ordem STYLOMMATOPHORA

Subordem ORTHURETHRA

Achatinellidae

Polygyridae

Oleacinidae

Sagdidae

Camaenidae

Bradybaenidae

Helminthoglyptidae

Helicidae

Ordem NOTASPIDEA

Umbraculidae

\section{Classe SCAPHOPODA}

Ordem DENTALIIDA

Dentaliidae

Classe BIVALVIA

Subclasse PTERIOMORPHIA

Ordem ARCOIDA

Arcidae

Glycymerididae

Ordem MYTILOIDA

Mytilidae

Pinnidae

Ordem PTERIOIDA

Subordem PTERIINA

Pteriidae

Pectinidae

Spondylidae

Subordem OSTREINA

Ostreidae

Subclasse PALAEOHETERODONTA

Ordem UNIONOIDA 
Hyriidae

Mycetopodidae

Subclasse HETERODONTA

Ordem VENEROIDA

Subordem ARCTICINA

Sphaeriidae

Corbiculidae

Chamidae

Montacutidae

Carditidae

Crassatellidae

Cardiidae

Tridacnidae

Mactridae

Mesodesmatidae

Solenidae

Tellinidae

Donacidae

Semelidae

Veneridae

Ordem MYOIDA

Subordem MYINA

Corbulidae

Erodonidae

Subordem PHOLADINA

Pholadidae

Teredinidae

Subclasse ANOMALODESMATA

Ordem PHOLADOMYOIDA

Clavagellidae

Classe CEPHALOPODA

Subclasse COLEOIDEA

Ordem SEPIOIDEA

Spirulidae

Ordem OCTOPODA

Subordem INCIRRATA

Argonautidae 
Vol. $7(1-2), 1990$

\section{REFERÊNCIAS BIBLIOGRÁFICAS}

MENEZES, J.A.B. \& THOMÉ, J.W. 1985. Nota preliminar sobre a Coleção Malacológica "Eliseo Duarte". Revista Brasileira de Zoologia, São Paulo, 3 (1):61-4.

MOORE, R.C., 1969. Treatise on Invertebrate Paleontology. The Zoological Society of America, Parte N, v. 1-3.

PICORAL, M. \& THOMÉ, J.W. 1987. Nota preliminar sobre a Coleção Malacológica "Eliseo Duarte", II. Revista Brasileira de Zoologia, São Paulo, 3 (8): 563-66.

PICORAL, M. et al. 1989. Nota preliminar sobre a Coleção Malacológica "Eliseo Duarte", III. Revista Brasileira de Zoologia, São Paulo, 6 (3):577-82.

RIOS, E.C. 1985. Seashells of Brazil. Rio Grande, Fundação Universidade do Rio Grande, Fundação Cidade do Rio Grande, 238 p., 102 est.

TAYLOR, D.W. \& SOHL, N.F. 1962. An outline of Gastropod Classification. Malacologia, Ann Arbor, 1 (1): 7-32. 